\title{
Optimization of mixed convection in a Lid-driven enclosure with a heat generating circular body.
}

\begin{abstract}
The physical model considered here is a lid-driven enclosure with bottom heating and top cooling conditions, and a heat generating circular body is placed at the center. The vertical walls of the cavity are kept thermally insulated, and the top lid moves at a constant speed. The steady two-dimensional governing equations for the physical problem are transformed in a dimensionless form with dimensionless governing parameters that decide the fluid flow and heat transfer characteristics in the system. The solution of these transport equations is obtained numerically with the finite element approach using the Galerkin method of weighted residuals. The parametric study has been carried out for variation of the heat generation parameters, the Reynolds numbers, solid-fluid thermal conductivity ratios as well as the Richardson numbers. The working fluid is assigned as air with a Prandtl number of 0.71 throughout the simulation. Results are presented in the form of streamlines, isotherms, average Nusselt number, bulk temperature, and drag force for the afore mentioned parameters. The numerical results indicate the strong influence of the mentioned parameters on the flow structure and heat transfer as well as average Nusselt number, average bulk temperature, and drag force. An optimum combination of the governing parameters would result in higher heat transfer and lower drag force.
\end{abstract}

Keyword: Optimization. 